\title{
Palatogingival Groove: A Cause for Periapical Infection
}

Ramesh Bharti, Anil Chandra, Aseem Prakash Tikku, Deeksha Arya

\begin{abstract}
The palatal groove is a developmental anomaly; when it is present in a tooth, it causes periodontal as well as endodontic problems. These grooves often present a challenge to the clinicians as diagnosing and treatment planning that requires an interdisciplinary treatment approach. This case report describes the successful collaborative management of a maxillary lateral incisor with an extensive palatal groove, using a combination of surgical endodontic therapy along with periodontal therapy.
\end{abstract}

Keywords: Developmental anomaly, Palatal groove, Root canal therapy.

How to cite this article: Bharti R, Chandra A, Tikku AP, Arya D. Palatogingival Groove: A Cause for Periapical Infection. Int J Prosthodont Restor Dent 2012;2(2):61-65.

Source of support: Nil

Conflict of interest: None

\section{INTRODUCTION}

Morphological defects in dental structure (e.g. dens invaginatus, talon cusp and the palatogingival groove) can be predisposing factors for the onset of inflammatory process in the periodontal and/or pulp tissues. These defects predominately affect the maxillary incisors. The palatogingival groove starts at the junction of the cingulum with one of the lateral marginal ridges and continue apically to the proximal surface of the root, ${ }^{1}$ possibly reaching the apex. ${ }^{2-4}$ Palatal grooves are quite variable not only in their depth but also in the distance and direction traversed down the root. ${ }^{1,5}$ Over the years, many other terms have been used to describe this anomaly, including palatogingival groove, ${ }^{1,6}$ developmental radicular anomaly, ${ }^{7}$ distolingual groove, ${ }^{8}$ radicular lingual groove, ${ }^{9,10}$ palatoradicular groove, ${ }^{6,11}$ radicular groove ${ }^{12}$ and cinguloradicular groove. ${ }^{2}$

Lee et $\mathrm{al},{ }^{5}$ speculated that the palatal groove represents an infolding of the enamel organ and Hertwig's epithelial root sheath. Unlike dens invaginatus, however, the infolding that occurs in the palatal groove is usually less extensive and creates an external defect that is situated adjacent to the gingival crevice. ${ }^{5}$ More recently, in a morphological analysis of these grooves, Ennes and Lara ${ }^{13}$ suggested that the palatal groove could be the result of an alteration of genetic mechanisms, rather than a dental germ folding.

Variation in the groove depth can make a possible communication with the pulp cavity. ${ }^{14,15}$ The funnel-like shape of the palatogingival groove promotes the accumulation of difficult-to-remove plaque and calculus, at times making proper cleaning by the patient, or even by the dentist, nearly impossible. ${ }^{7-9,15}$ Periodontal disease can jeopardize the pulp depending on the groove's depth which may even reach the root apex. ${ }^{2}$. Current clinical treatment can correspond to that for inflammatory periodontal diseases. ${ }^{2,3,7,9,15-17}$ In some cases, the palatogingival groove can be seen in periapical radiographs as a fine parapulpal radiolucent line. ${ }^{1,3,4,8,7}$ The prognosis of pulp diseases and/ or periapical inflammation in the presence of a palatogingival groove is not very favorable and depends in part on the groove's extension, the depth of the groove and the relation of the groove to the pulp cavity. ${ }^{2,3,8,17,18}$ Treatment modalities have ranged from extraction to cavity preparation of the defect and restoration with amalgam; in the majority of cases, endodontic treatment is necessary. ${ }^{19}$

Palatal grooves on maxillary incisors often present a diagnostic and treatment planning dilemma. While frequently associated with periodontal pockets and bone loss, pulpal necrosis of these teeth may precipitate a combined endodontic-periodontic lesion. This article presents a case of a lateral incisor anatomically complicated with palatogingival groove and pulpal involvement along with labially draining sinus. The management of the tooth was done with an interdisciplinary approach.

\section{CASE REPORT}

A healthy 40-year-old female was referred to the department of conservative dentistry and endodontics with a sinus tract and periapical radiolucency associated with her maxillary right lateral incisor. The patient was unaware of any previous trauma to the maxillary anterior region and had no history of pain in that area. Clinically, the patient presented with a facial parulis in the alveolar mucosa adjacent to maxillary right lateral incisor that had been present for approximately 3 months (Fig. 1). Maxillary right lateral incisor did not respond to pulp testing with a Kerr Electric Pulp Tester (SybronEndo, Orange, CA, USA), while the adjacent teeth responded normally to pulp test. The patient had no significant tenderness to percussion or palpation in the maxillary anterior area and probing depths were normal, except for a narrow $5 \mathrm{~mm}$ pocket on the palatal aspect associated with a palatal groove (Fig. 2). Periapical radiographs revealed an extensive periradicular radiolucency involving the apical two-third of the root on the maxillary right lateral incisor (Fig. 3). A diagnosis of endodontic periodontal lesion with palatogingival groove was inferred. 


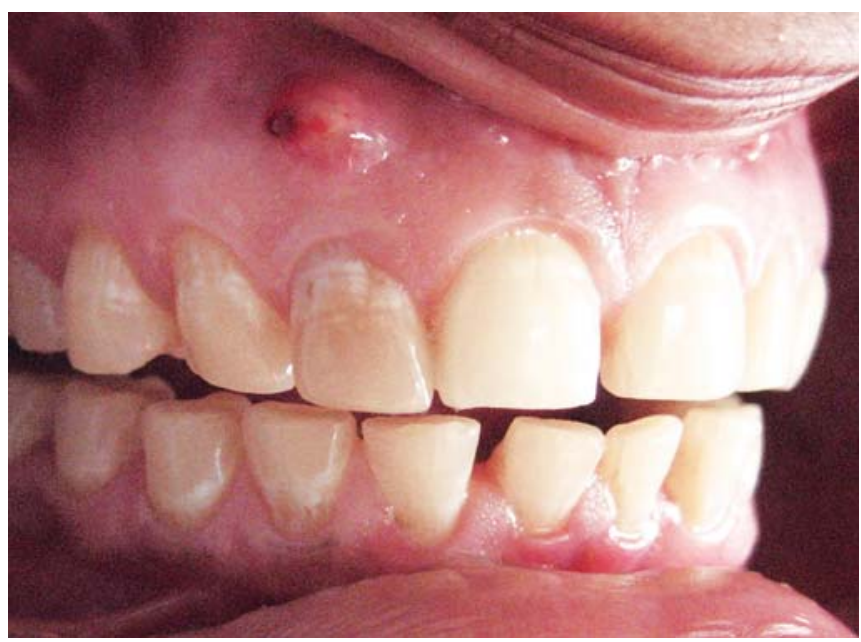

Fig. 1: Photograph demonstrating the sinus

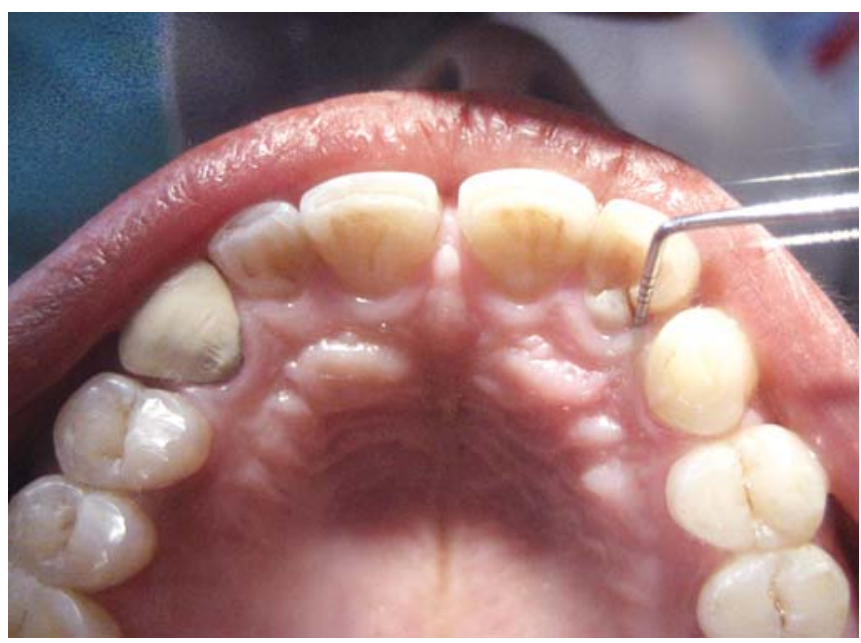

Fig. 2: Palatogingival groove in maxillary right lateral incisor

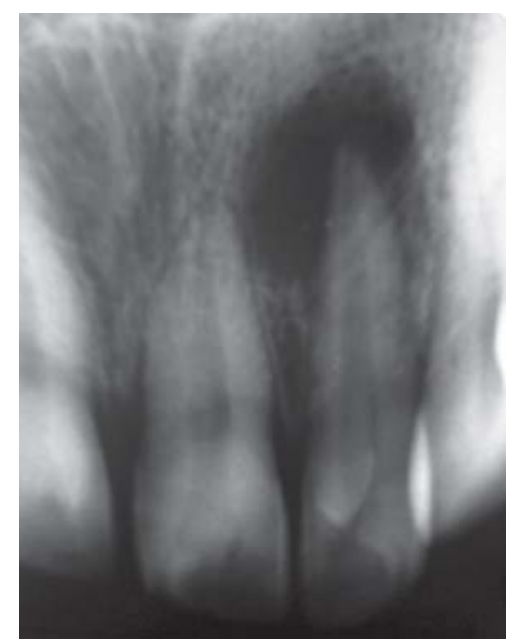

Fig. 3: Periapical radiograph showing periradicular radiolucency associated with maxillary right lateral incisor one vertically oriented radiolucent line is also evident within tooth

An interdisciplinary treatment plan was formulated for the patient.

After prophylaxis and removal of localized calculus, endodontic access was started under rubber dam isolation.
Cleaning and shaping of the canal were carried out by using the crown-down technique up to an apical file size 60 . The tooth was obturated by using gutta-percha and $\mathrm{AH}$ plus sealer (Dentsply Maillefer, Ballaigues, Switzerland) by using lateral condensation technique (Fig. 4). During the periodontal phase of the therapy, a surgical flap was raised from the palatal aspect and the palatogingival groove was isolated to its most apical extent (Fig. 5). Thorough scaling and root planning was performed over the groove to remove the bacteria which might have colonized there. The diseased granulation tissue was curetted (with Gracey Curette number 1, 2 and 5, 6; Hu-Friedy Manufacturing Co, Chicago, IL, USA) to leave the soft tissue more conducive to regeneration. A chemical conditioning of the groove was performed by using $10 \%$ polyacrylic acid, and glass ionomer cement (GIC) type I (Fuji I; GC Corporation, Tokyo, Japan) was applied into the defect (Fig. 6). The area was kept isolated from blood and tissue fluids during the setting of the cement by using local hemostatic gelatin sponge (Pfizer Inc., New York, NY, USA). After palatal groove management, facial flap was

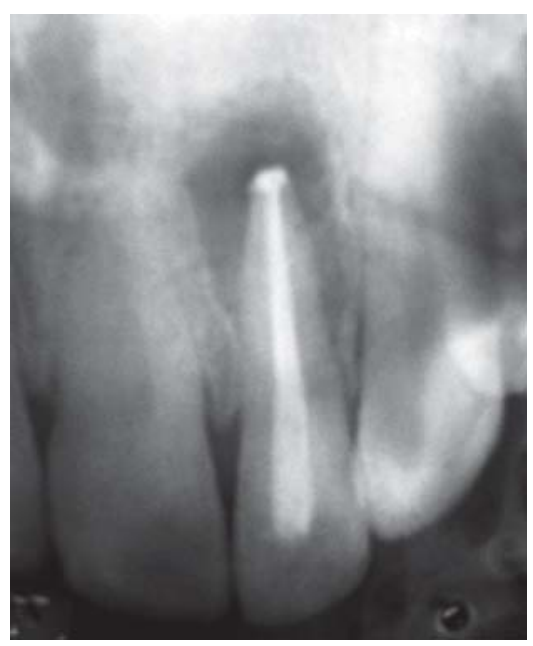

Fig. 4: Periapical radiograph showing well-obturated canal

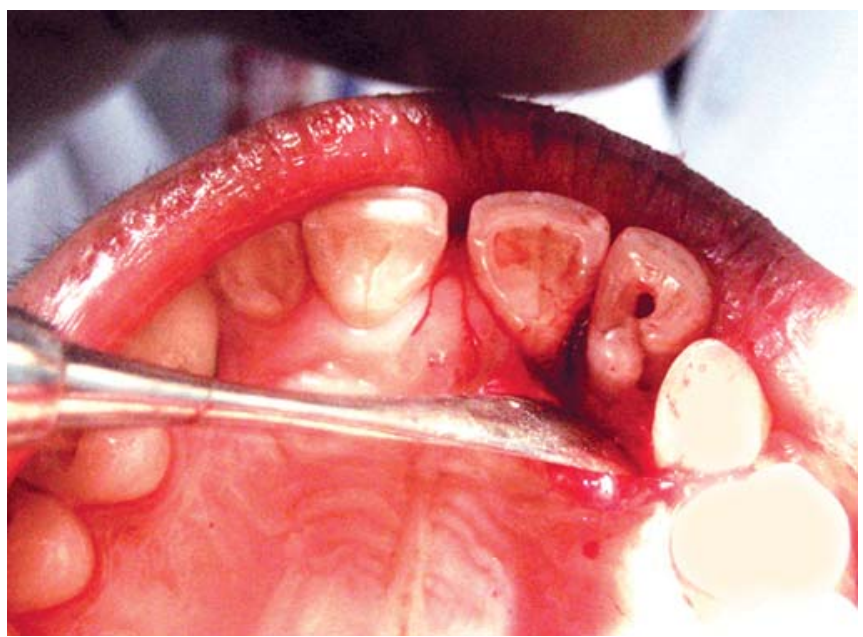

Fig. 5: The palatogingival groove isolated after raising surgical flap from the palatal aspect 
raised to access the facial bony fenestration of the cortical plate (Fig. 7). The soft tissue present in the bony defect was removed and given for histopathological examination, and the root surfaces exposed to the defect were placed with an ultrasonic scaler (Odontoson, Odonto-Wave, Fort Collins, CO, USA) and hand curettes. Then flap was approximated and sutured (Fig. 8). Patient was instructed about postsurgery precautions and maintenance protocol, which included rinsing with $0.12 \%$ solution of chlorhexidine twice a day for 5 weeks. During this period, he was recalled once a week for professional cleaning teeth. Sutures were removed on 7th day after the surgery.

The patient was recalled for professional teeth cleaning and reinforcement of self-performed oral hygiene measures at 1-month intervals for 1 year. Three months after the surgery, the probing depth around the palatal aspect of the lateral incisor did not exceed $2 \mathrm{~mm}$ and periapical healing was excellent (Fig. 9). After 1 year, no recession of the interdental papilla or palatogingival margin was noticed (Figs 10 and 11).

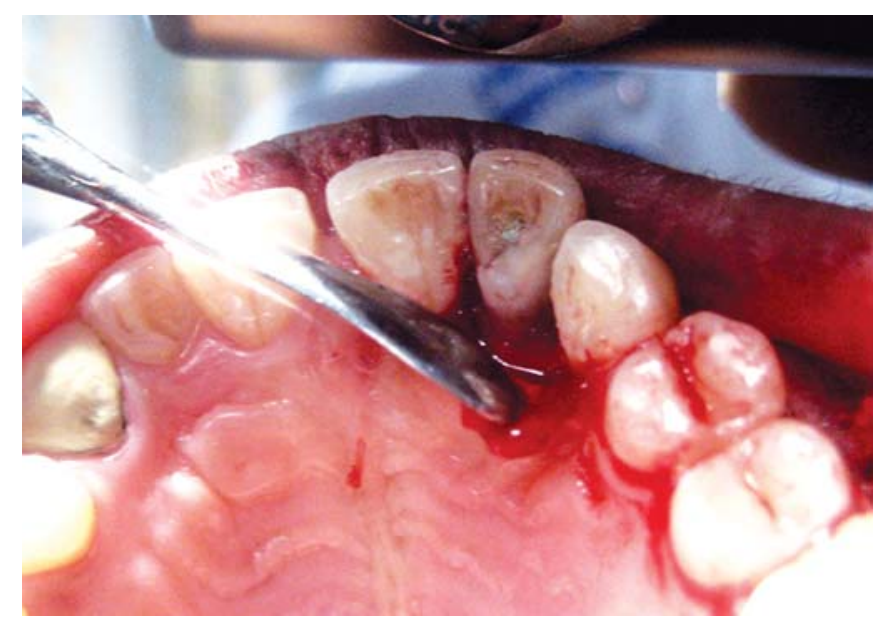

Fig. 6: The palatogingival groove restored with glass ionomer cement

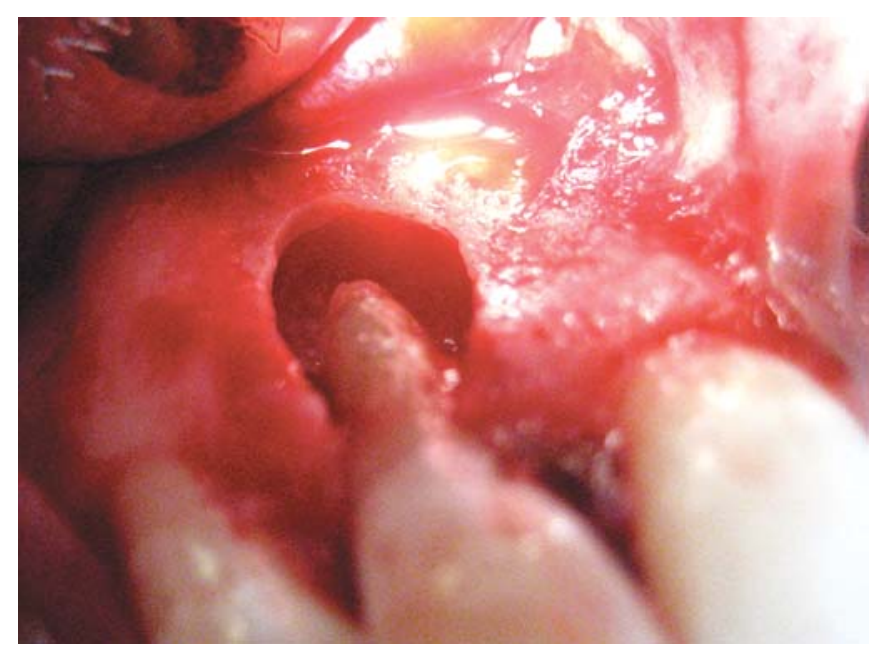

Fig. 7: Raised surgical flap showing large bony cavity after removing granulation tissue

\section{DISCUSSION}

Developmental infoldings in dental structure (dens invaginatus, talons cusp, palatogingival groove) can be the predisposing factors for the onset of inflammatory reactions

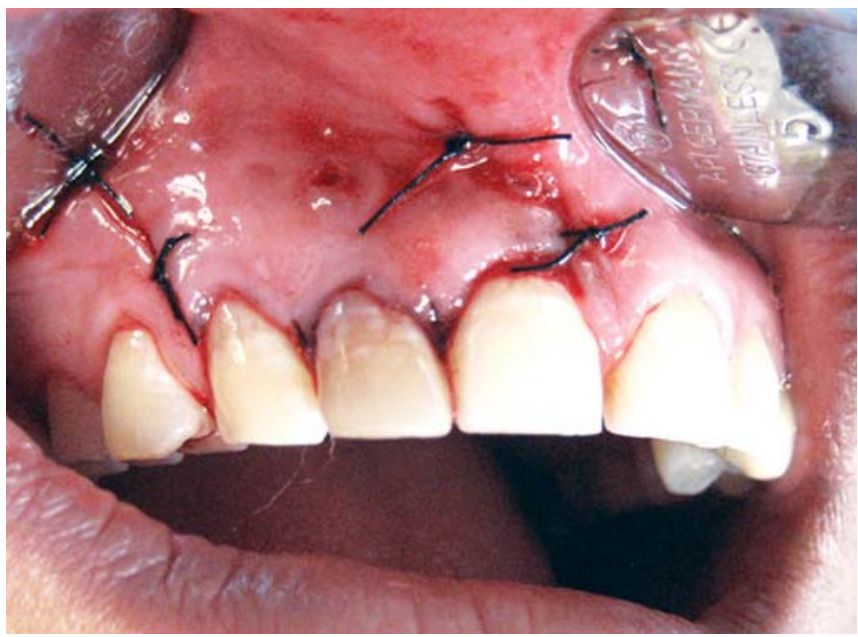

Fig. 8: Sutured flap in its position

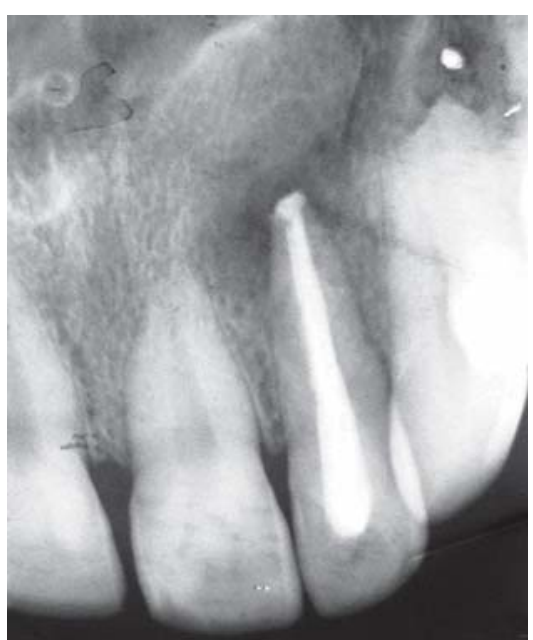

Fig. 9: Radiograph showing healing after 3 months

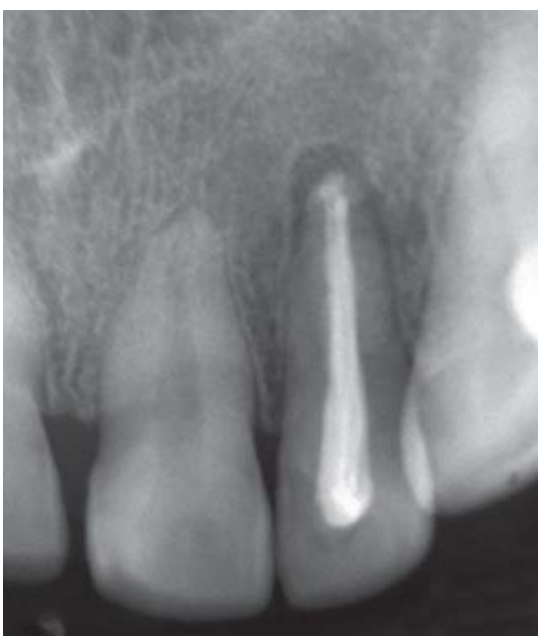

Fig. 10: Radiograph showing healing after 1 year 


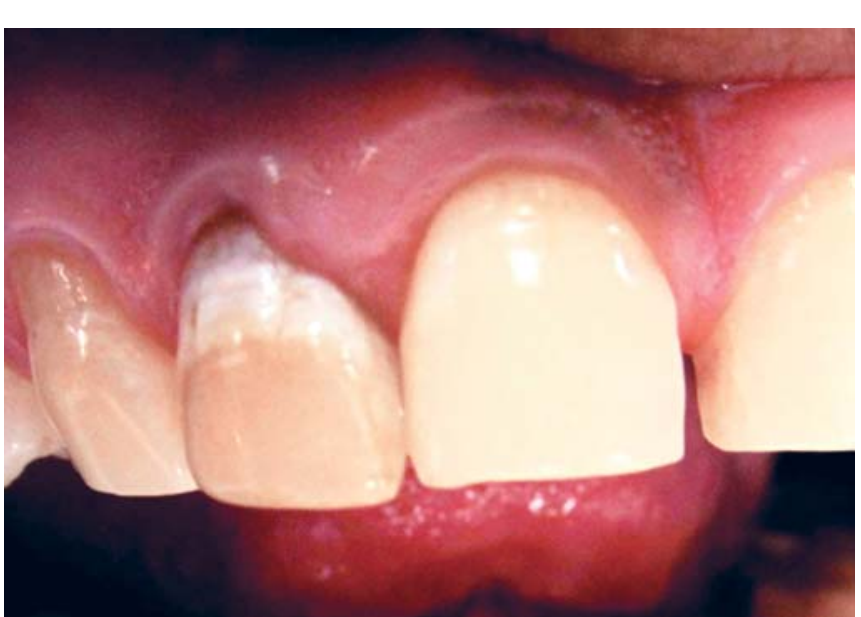

Fig. 11: Photograph after 1 year showing excellent gingival health

in pulp and/or periodontal tissues. Several studies have been conducted in order to determine the prevalence of palatogingival groove. The prevalence rate has been reported to be approximately 2.8 to $8.5 \%$. According to a study done by Lara, ${ }^{1}$ macroscopic analysis of teeth revealed that palatogingival groove most commonly occur on the lingual surface of maxillary lateral incisor.

According to a study done by Kagon on staining with methylene blue and then observing under microscope, he found that, out of 1786 maxillary lateral incisors, 100 exhibited palatogingival groove. Out of these 100 grooves, 77 originated in cingulum, 15 in lateral fossa, 7 at cementoenamel junction and 1 originated on root proceeding apically, 13 were located on the mesial side, 25 on distal, 62 on midpalatal area of lingual surface. In 37\% cases, cementoenamel junction was displaced apically or incisally in the area where palatogingival groove crossed the junction. $^{5}$

Gao et al ${ }^{14}$ utilized scanning electron microscope to examine palatogingival groove in 14 teeth to study the depth of groove. The depth of grooves varied and was deepest in the coronal half of the root. In the deepest groove, the remaining dentin thickness was only $360 \mu \mathrm{m}$ and 1-3 accessory foramina were found (15-200 $\mu \mathrm{m}$ in diameter) at the base or lateral wall of groove.

Recognizing palatogingival groove as the initiators of pathology can be difficult. Patients may present with pulpal involvement in teeth that have no caries or history of trauma. ${ }^{9,19}$ Conversely, patient may demonstrate periradicular abscess formation in teeth with vital pulp. Several authors have reported cases in which patients with pathological involvement received delayed or improper treatment, since palatogingival groove was not diagnosed. ${ }^{3,9,19}$
Not all the patients with pathological palatogingival groove are symptomatic. However, with careful observation, they can generally be diagnosed. Grooves originating in the cingulum are clinically visible. The gingiva adjacent to groove is often edematous, erythematous or cyanotic. Periodontal probing is recommended for such patients. Patients with palatogingival groove have deep isolated 'tubular shaped' periodontal pockets that frequently bleed. Grooves may be visible on intraoral periapical radiograph as one or many dark lines extending along the length of root parallel to or superimposed over root canal.

The prognosis of teeth affected by this anomaly depends on the location, depth, extension of the groove and the extent of periodontal destruction. Teeth with shallow grooves located entirely on the crown might be corrected with odontoplasty along with periodontal therapy. However, when groove is deep with advanced periodontal or and pulpal destruction, treatment becomes complex. The success of the treatment depends on complete eradication of inflammatory stimulus by elimination of groove and encouraging patient to maintain proper hygiene.

For sealing, the palatogingival groove several materials have been used like composite, amalgam, GIC and mineral trioxide aggregate (MTA). Disadvantage with MTA is that it might get washed off in transgingival defect. GIC was chosen for the present case because it adheres chemically to the tooth, has adequate sealing ability ${ }^{20,21}$ and promotes epithelial and connective tissue attachment. ${ }^{22 .}$

So, the rationale behind current treatment plan was to restore deep palatogingival groove. To eliminate irritant stimulus and to prevent bacterial colonization, the groove was cleaned and sealed to prevent recurrence. For achieving these results combined endodontic and periodontal treatment was performed.

After the collaborative management of the palatogingival groove and periradicular pathology of this case, healing was evident both clinically and radiographically. So, complex interdisciplinary approach provides much hope for such type of cases.

\section{CONCLUSION}

An association between palatal groove and periodontal complications is relatively rare. Yet when a palatal groove is present, diagnosing a pulpal or localized periodontal lesion can sometimes pose a dilemma for the general dentist because these radicular grooves act as 'funnels' aiding microbial plaque accumulation, leading to gingivitis, localized periodontitis and even pulpal necrosis. However, when a palatal groove does occur and a timely diagnosis is 
made, there are recognized ways to manage the situation and, in many cases, the involved tooth or teeth can be saved.

\section{CLINICAL SIGNIFICANCE}

Thorough examination of the morphological anomalies associated with the lateral and central incisors, in particular, aided by appropriate diagnostic aids can lead to accurate diagnosis and management of a tooth or teeth with a palatoradicular groove.

\section{REFERENCES}

1. Lee KW, Lee EC, Poon KY. Palato-gingival grooves in maxillary incisors. A possible predisposing factor to localised periodontal disease. Br Dent J 1968;124(1):14-18.

2. Assaf ME, Roller N. The cingulo-radicular groove: Its significance and management- - two cases reports. Comp Contin Educ Dent 1992;13:94-100.

3. Robison SF, Cooley RL. Palatogingival groove lesions: Recognition and treatment. Gen Dent 1988;36(4):340-42.

4. Walker RT. The disto-palatal groove in maxillary incisors-a predisposing factor in periodontal disease. J R Nav Med Sew 1976;62:30-32.

5. Kogon SL. The prevalence, location and conformation of palatoradicular grooves in maxillary incisors. J Periodontol 1986;57:231-34.

6. Withers JA, Brunsvold MA, Killoy WJ, Rahe AJ. The relationship of palato-gingival grooves to localized periodontal disease. J Periodontol 1981;52:41-44.

7. Simon JH, Glick DH, Frank AL. Predictable endodontic and periodontic failures as a result of radicular anomalies. Oral Surg Oral Med Oral Pathol 1971;31:823-26.

8. Everett FG, Kramer GM. The disto-lingual groove in the maxillary lateral incisor — a periodontal hazard. J Periodontol 1972;43:352-61.

9. August DS. The radicular lingual groove: An overlooked differential diagnosis. J Am Dent Assoc 1978;96:1037-39.

10. Meister F Jr, Keating K, Gerstein H, Mayer JC. Successful treatment of a radicular lingual groove: Case report. J Endod 1983;9:561-64.

11. Hou GL, Tsai CC. Relationship between palato-radicular grooves and localized periodontitis. J Clin Periodontol 1993;20:678-82.

12. Pecora JD, Sousa Neto, Santos TC, Saquy PC. In vitro study of the incidence of radicular grooves in maxillary incisors. Braz Dent J 1991;2:69-73.

13. Ennes JP, Lara VS. Comparative morphological analysis of the root developmental groove with the palato-gingival groove. Oral Dis 2004;10:378-82.

14. Gao ZR, Shi JN, Wang Y, Gu PI. Scanning electron microscopic investigation of maxillary lateral incisors with a radicular lingual groove. Oral Surg Oral Med Oral Pathol 1989; 68:462-65.
15. Santa Cecilia M, Lara VS, Moraes IG. The palato-gingival groove. A cause of failure in root canal treatment. Oral Surg 1998;85:94-98.

16. Paul BF. Surgical management of the palato-radicular groove and associated periodontal lesion: A case presentation. Pract Periodont Aesth Dent 1993;5:25-28.

17. Brunsvold MA. Amalgam restoration of a palatogingival groove. Gen Dent1985;33:244-46.

18. Jeng JH, Lu HKJ, Hou LT. Treatment of an osseous lesion associated with a severe palato-radicular groove: A case report. J Periodontol 1992;63:708-12.

19. Benenati FW. Maxillary second molar with two palatal canals and a palatogingival groove. J Endodont 1985;11:308-10.

20. Maldonado A, Swartz ML, Philips RW. An in vitro study of certain properties of glass ionomer cement. J Am Dent Assoc 1978;96:785-91.

21. Vermeersch G, Lelop G, Delmee M, Vreven J. Antibacterial activity of glass ionomer cements, compomers and resin cements: Relationship between acidity and and material setting phase. J oral rehabil 2005;32:368-74.

22. Dragoo MR. Resin ionomer and hybrid ionomer cements: Part II-human clinical and histologic wound healing responses in specific periodontal lesions. Int J Periodontics Restorative Dent 1997; $17: 75-87$

\section{ABOUT THE AUTHORS}

\section{Ramesh Bharti}

Assistant Professor, Department of Conservative Dentistry and Endodontics, Faculty of Dental Sciences, King George's Medical University, Lucknow, Uttar Pradesh, India

Correspondence Address: Flat No.-805, Doctor's Flat, TG Hostel Campus, Khadra, Sitapur Road, Lucknow-226 003, Uttar Pradesh India, Phone: +91-522-2743556, +91-9935724723, Fax: +91-5222254555, e-mail: r_bharti14@yahoo.com

\section{Anil Chandra}

Professor, Department of Conservative Dentistry and Endodontics Faculty of Dental Sciences, King George's Medical University Lucknow, Uttar Pradesh, India

\section{Aseem Prakash Tikku}

Professor, Department of Conservative Dentistry and Endodontics Faculty of Dental Sciences, King George's Medical University Lucknow, Uttar Pradesh, India

\section{Deeksha Arya}

Assistant Professor, Department of Prosthodontics, Faculty of Dental Sciences, King George’s Medical University, Lucknow, Uttar Pradesh India 